\title{
Facile Tuning of Superhydrophobic States with Ag Nanoplates
}

\author{
Yugang $\operatorname{Sun}^{1}(\bowtie)$ and Rui Qiao ${ }^{2}$ \\ ${ }^{1}$ Center for Nanoscale Materials, Argonne National Laboratory, 9700 South Cass Avenue, Argonne, IL 60439, USA \\ ${ }^{2}$ Department of Mechanical Engineering, College of Engineering and Science, Clemson University, Clemson, SC 29634, USA \\ Received: 22 July 2008/ Revised: 15 August 2008/Accepted: 15 August 2008 \\ CTsinghua Press and Springer-Verlag 2008. This article is published with open access at Springerlink.com
}

\begin{abstract}
GaAs wafers have been decorated with $\mathrm{Ag}$ nanoplates through direct galvanic reaction between aqueous $\mathrm{AgNO}_{3}$ solutions and $\mathrm{GaAs}$, resulting in Ag nanoplate/ GaAs composite surfaces with varying hydrophobocity after the Ag nanoplates were coated with self-assembled monolayers of alkyl thiol molecules. By carefully controlling the reaction conditions, such as growth time and concentration of the $\mathrm{AgNO}_{3}$ solution, the size, thickness, and surface roughness of the individual Ag nanoplates can be tuned in order to produce different topographic structures and roughness of the composite surfaces, which in turn influences the hydrophobicity of the surfaces. The as-synthesized composite surfaces have been found to exhibit various levels of hydrophobicity and different wetting states such as the Wenzel wetting state, Cassie impregnating wetting state, and Cassie nonwetting state. The relationship between surface structure and hydrophobic state is also discussed.
\end{abstract}

\section{KEYWORDS}

Superhydrophobic, Cassie, Wendel, Ag nanoplates, GaAs, self-assembled monolayers

\section{Introduction}

Controlling the hydrophobicity of solid surfaces has recently become an active research area due to its technological importance in a wide range of applications such as anti-sticking, anti-contamination, self-cleaning, and oil/water separation [1-11]. In particular, it is desirable to create superhydrophobic surfaces that exhibit apparent contact angles larger than $150{ }^{\circ} \mathrm{C}$. Although it is well known that the hydrophobicity of a surface can be enhanced by coating the surface with thin layers of low surface energy materials (e.g., long-chain alkyl thiol molecules for precious metals, or fluoroalkylsilane molecules for $\mathrm{Si}$ ), achieving superhydrophobicity through this strategy is difficult or even impossible
$[12,13]$. An alternative approach is to create artificial surfaces by mimicking natural superhydrophobic surfaces, such as lotus leaves and petals of red roses or sunflowers, which exhibit great roughness [14, 15]. Wenzel, Cassie, and Baxter pioneered the theoretical studies of such surfaces $[16,17]$. They concluded that the surface roughness (in addition to low surface energy) plays a vital role in determining the surface hydrophobicity, and deduced equations to describe the relationship between the Wenzel roughness, the Cassie-Baxter surface fractions, and the apparent static contact angle. Since then, a large number of physical approaches (e.g., lithographic patterning and etching, molding, and imprinting) have been used to create roughness on surfaces in order to achieve superhydrophobicity [11, 15, 18-22]. Alternatively,

Address correspondence to ygsun@anl.gov 
inducing surface roughness by direct deposition of micro/nanostructures of different materials (e.g., metals [23-29], polymers [19, 30-34], polyelectrolytes [35], oxides [1, 36-39], or carbon nanotubes [3, 4042]) on substrates through chemical reactions and/or assembly processes has also been shown to lead to superhydrophobicity.

These efforts have yielded a more detailed picture of the superhydrophobicity of composite surfaces with different roughness. For example, it has been found that as-fabricated surfaces can exhibit variant hydrophobic states, including the Wenzel state, Cassie superhydrophobic state, "Lotus" state, transitional superhydrophobic state between Wenzel and Cassie states, "Gecko" state, and Cassie impregnating wetting state, as defined by Jiang et al. [15, 43]. Although these six states have all been reported in the literatures, it is generally accepted that the Wenzel wetting state and the Cassie composite state (where water droplets sit partially on the solid surface and partially on air underneath the droplets) represent the two basic hydrophobic states and other states can be classified into one or other of these categories. For example, the "Lotus" state (also called the Cassie nonwetting state) is a special Cassie state with very high contact angle and extremely low contact angle hysteresis [14]. In addition, the Cassie nonwetting state can be irreversibly converted to the Wenzel wetting state by applying external pressure [44]. The intermediate state during this transition may be the Cassie impregnating wetting state, which exhibits high contact angle and large contact angle hysteresis (and thus strong adhesion between water droplets and surfaces) [15, 34, 45]. Despite this progress, understanding of the hydrophobicity of rough surfaces remains limited. For example, the classification of the hydrophobic states outlined above is still under debate and quantitative understanding of the relationship between the apparent contact angles in these states and surface structures (or topographic geometries) is rudimentary $[46,47]$. Producing composite surfaces with a range of superhydrophobic behavior through simple approaches also remains difficult.

In this article, we study the hydrophobic properties of a new class of composite surfaces, i.e., GaAs substrates covered with high aspect ratio (i.e., having a large ratio of diameter to thickness) Ag nanoplates, which are grown through direct reaction between pure aqueous solutions of $\mathrm{AgNO}_{3}$ and the GaAs substrates themselves [48-51]. The as-grown Ag nanoplates are coated with self-assembled monolayers (SAMs) of 1-hexadecanethiol (HDT) molecules in order to decrease the surface energy of Ag. Remarkably, wettability of the resulting Ag nanoplate/GaAs composite surfaces can be easily tuned between the Wenzel wetting state, the Cassie nonwetting state, and the Cassie impregnating wetting state by controlling the dimensions and nanoscale surface roughness of individual nanoplates, by appropriate variation of reaction conditions.

\section{Experimental}

Ag nanoplates were grown using the approaches described elsewhere [48-51]. n-type GaAs wafers with dopant (Si) concentration of $\sim 1 \times 10^{18} \mathrm{~cm}^{-3}$ (AXT, Fremont, CA) were cut into $\sim 1 \mathrm{~cm} \times 1 \mathrm{~cm}$ square pieces. The small GaAs shards were soaked in a $2 \%$ hydrofluoric acid (HF) aqueous solution (Fisher Scientific, Pittsburg, PA) for 5 min to remove native oxides from their surfaces. Caution: personal protective equipment is required to handle $\mathrm{HF}$, which is highly corrosive toward tissues, and bones in particular. The shards were then thoroughly rinsed with deionized (DI) water followed by drying with a gentle $\mathrm{N}_{2}$ flow. The $\mathrm{AgNO}_{3}$ solutions with different concentrations were freshly prepared by dissolving appropriate amounts of $\mathrm{AgNO}_{3}$ (Aldrich, Milwaukee, WI) in DI water. The reactions were carried out in an ambient environment in a conventional chemistry laboratory and at room temperature. In a typical reaction, a droplet $(\sim 20 \mu \mathrm{L})$ of $\mathrm{AgNO}_{3}$ solution was quickly delivered to the central area of the flat surface of a cleaned GaAs shard with a micropipettor. The shard was covered with a black cap to eliminate the effect of light illumination (although the effect is minor) and evaporation of water during the reaction. The reaction was terminated by immersing the GaAs wafer in a large volume $(\sim 1 \mathrm{~L})$ of water to remove excess $\mathrm{AgNO}_{3}$. Finally, the wafer was rinsed with DI water and dried with a gentle $\mathrm{N}_{2}$ flow. The 
as-synthesized fresh samples were then soaked in a $5 \mathrm{mmol} / \mathrm{L}$ ethanolic solution of 1-hexadecanethiol (Aldrich) for $1 \mathrm{~h}$ followed by thorough rinsing with ethanol and drying with a gentle $\mathrm{N}_{2}$ flow. The resulting thiol-modified samples were used for hydrophobic measurements.

All the measurements of contact angles and sliding angles were carried out on a First Ten Ångstroms system (Portmouth, VA). In a typical measurement, a drop ( $4 \mathrm{mg})$ of DI water was delivered on an Ag nanoplate/ GaAs composite substrate horizontally sitting on a stage by slowly pushing the plug of a syringe located close to the top surface of the substrate. After the water droplet stabilized on the composite surface, a photograph was taken by the camera and the contact angles were determined by drawing the tangent of the water/air interface and the line that represents the nominal surface of the composite substrate. The sliding angle was measured by slowly tilting the substrate to a certain angle at which the water droplet started to slide off the composite surface.

\section{Results}

Figures 1 (a)-(d) present the scanning electron microscopy (SEM) images of Ag nanoplates grown on highly doped n-type single crystalline GaAs wafers through simple galvanic reaction with a neutral aqueous solution of $\mathrm{AgNO}_{3}$ with concentration of $2 \mathrm{~mol} / \mathrm{L}$ :

$$
\begin{aligned}
& 12 \mathrm{AgNO}_{3}+2 \mathrm{GaAs}+6 \mathrm{H}_{2} \mathrm{O} \rightarrow \\
& 12 \mathrm{Ag}+\mathrm{Ga}_{2} \mathrm{O}_{3}+\mathrm{As}_{2} \mathrm{O}_{3}+12 \mathrm{HNO}_{3}
\end{aligned}
$$

This room-temperature reaction produces highquality Ag nanoplates, with dimensions which depend on the growth time, on the GaAs substrates [51]. For example, their average sizes (i.e., the lengths of the orthographic projections of individual nanoplates along their longitudinal axes on the surfaces of the substrates) increase from 110 to $320 \mathrm{~nm}$ when the growth time increases from 15 to $90 \mathrm{~s}$ (Fig.

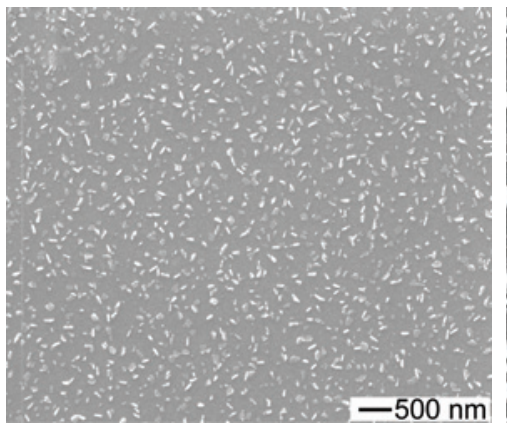

(a)

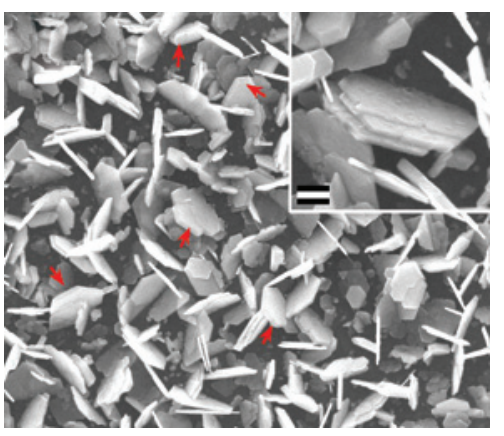

(c)

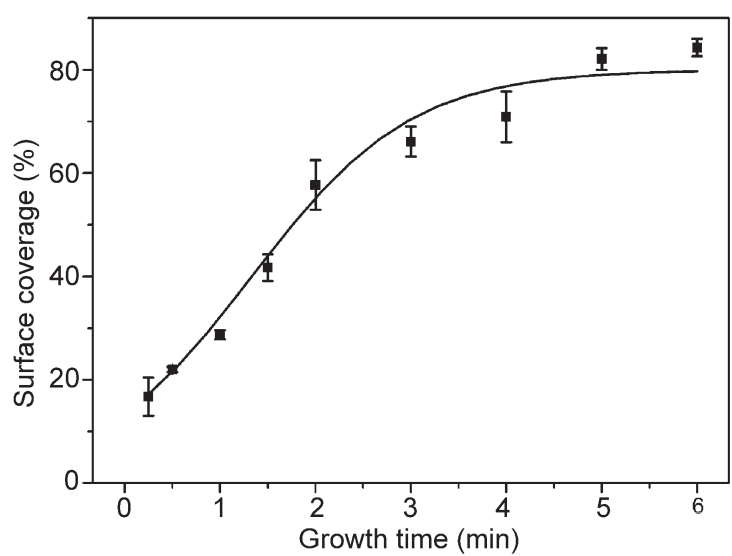

(e)

(b)

(d)
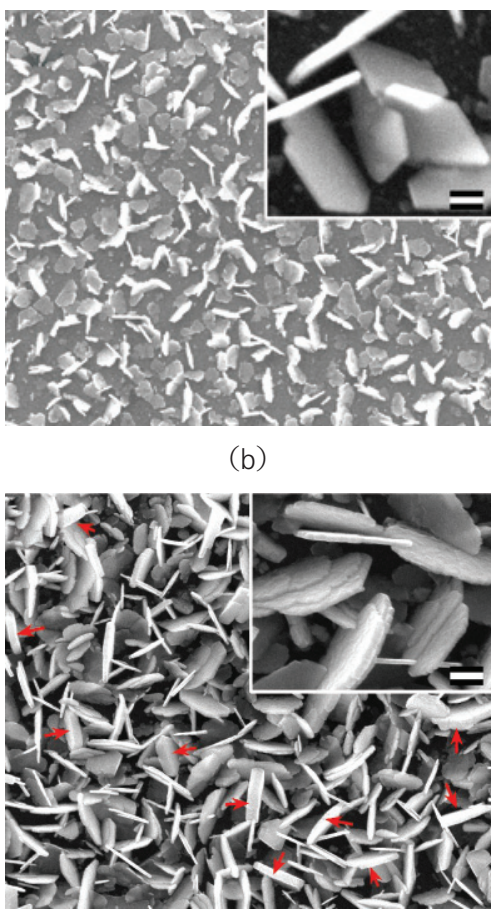

Figure 1 (a)-(d) SEM images of Ag nanoplates grown on GaAs substrates by reaction with a $2 \mathrm{~mol} / \mathrm{L} \mathrm{AgNO}_{3}$ solution for different times: (a) $15 \mathrm{~s}$; (b) $1.5 \mathrm{~min}$; (c) $4 \mathrm{~min}$; and (d) $6 \mathrm{~min}$. (e) Dependence of surface coverage of the Ag nanoplates on the GaAs substrates on the growth time. The scale bar in (a) also applies to (b)-(d) and the scale bars in the insets represent $200 \mathrm{~nm}$

1(a) versus Fig. 1(b)). The thickness of the nanoplates formed at short growth times $(<3 \mathrm{~min})$ is essentially constant with an average size of $22 \mathrm{~nm}$ and their surfaces are flat (as highlighted in the inset of Fig. 1(b)). Most plates protrude out of the surfaces of the GaAs substrates although their orientations fluctuate from vertical to almost horizontal with respect to the GaAs substrates. Deposition of Ag nanoplates in this fashion increases the macroscopic roughness (on the micrometer scale) of the substrates. Observation of 
cross-sectional samples (Fig. S-1 in the ESM) shows that each Ag nanoplate contacts the GaAs lattice of the growth substrate only through a small defect site (e.g., the tiny post highlighted in the circle in Fig. S-1 in the Electronic Supplementary Material (ESM)). The edge of each nanoplate, excluding the contact point, is separated by a very thin layer of oxides of $\mathrm{Ga}$ and As formed during reaction (1). When the reaction is continued for more than $3 \mathrm{~min}$, some adjacent nanoplates tend to polymerize into oligomers (typically dimers and trimers) followed by fusion into thicker nanoplates [51]. Figures 1(c) and 1(d) give the SEM images of the samples formed at 4 and $6 \mathrm{~min}$, respectively, highlighting the existence of oligomers (see the insets) and thick plates (indicated by the arrows). Meanwhile, the concentration of $\mathrm{AgNO}_{3}$ drops too much to completely support the anisotropic growth of nanoplates at growth time of $>$ $3 \mathrm{~min}$, and the formation of nanograins on the basal surfaces of nanoplates is observed (see the insets in Figs. 1(c) and 1(d)). Decoration with nanograins leads to an increase in the microscopic roughness (on the nanometer scale) of individual nanoplates. Apparently, the surface coverage of Ag nanoplates on a GaAs substrate, which is shortened as "surface coverage" hereafter, increases with the size of the nanoplates. The growth of Ag nanoplates results in an increase in macroscopic roughness of the Ag nanoplate/GaAs composite surfaces. The surface coverage is estimated by integrating the bright areas (corresponding to the Ag nanoplates) of an SEM image and calculating integrated bright area as a percentage of the whole area of the SEM image. Figure 1(e) plots the dependence of the surface coverage on the growth time. The curve indicates that the size of Ag nanoplates increases rapidly in the early stages (i.e., growth times $<3 \mathrm{~min}$ ), resulting in a fast increase in the surface coverage. At growth time longer than $3 \mathrm{~min}$, the surface coverage only slightly increases with growth time because further reaction only marginally increases the sizes of the nanoplates. However, the microscopic roughness of individual nanoplates increases significantly at longer growth time (insets of Figs. 1(c) and 1(d)).

The Ag nanoplate/GaAs composite surfaces exhibit very interesting wetting behavior after modification with SAMs of HDT molecules. Figure 2 plots the apparent static contact angles (which are abbreviated as "contact angle" in all figures for convenience) and sliding angles as a function of surface coverage of the Ag nanoplates. Here, the sliding angle is defined as the critical angle at which a water droplet with a mass of $\sim 4 \mathrm{mg}$ begins to slide down an inclined surface. It can be clearly seen that the samples formed at longer growth time are more hydrophobic than the samples formed at shorter time. The contact angle increases dramatically from $\sim 105^{\circ}$ to $\sim 150^{\circ}$ when the surface coverage increases from $\sim 16 \%$ to $\sim 30 \%$ (i.e., the corresponding growth time increases from $15 \mathrm{~s}$ to $\sim 60 \mathrm{~s}$ ) (Zone I in Fig. 2). The contact angle of a water droplet on the substrate formed after $15 \mathrm{~s}$ is close to that of a water droplet on a flat GaAs wafer or a flat Ag film modified with SAMs of HDT molecules (Fig. S-2 in the ESM). This indicates that the wettability of $\mathrm{Ag}$ nanoplate/GaAs composite surfaces with low surface coverage $(<15 \%)$ is dominated by the GaAs surfaces because the roughness generated by the small $\mathrm{Ag}$ nanoplates is negligible. As the surface coverage increases in

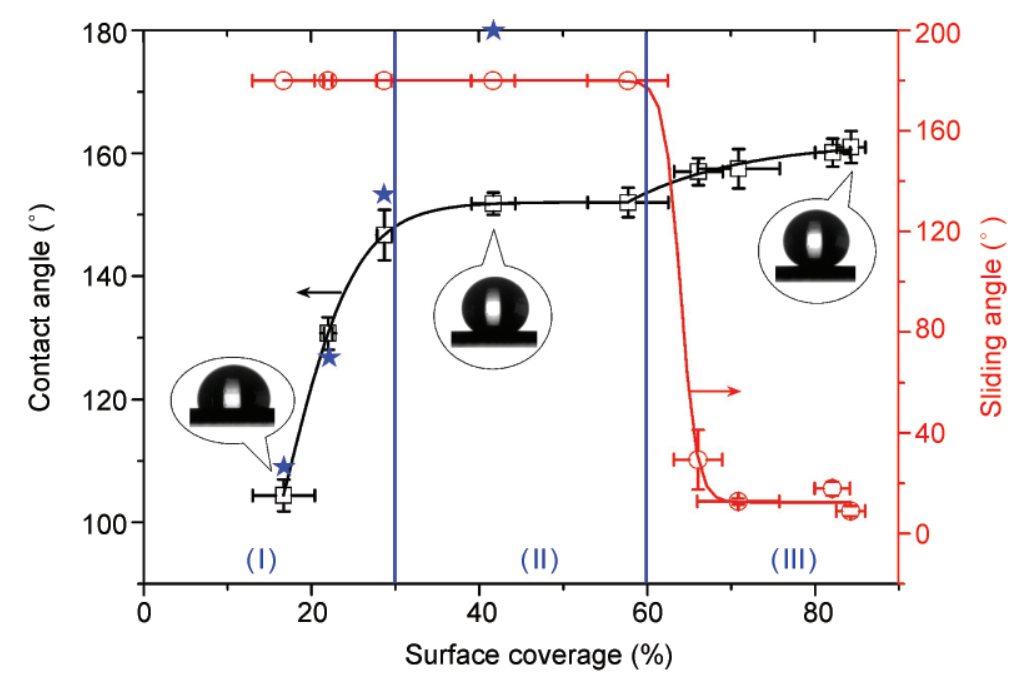

Figure 2 Relationship between apparent contact angles and sliding angles of water droplets on the surfaces shown in Fig. 1 and the surface coverage represented in Fig. 1(e). The insets show optical images of water droplets on the as-synthesized surfaces. The solid blue stars indicate the contact angles calculated according the Wenzel equation (Eq. (2)). Three zones (i.e., Zone I, Zone II, and Zone III) are separated by vertical blue lines in order to highlight the three different hydrophobic states 
the range $\sim 30 \%-60 \%$, the macroscopic roughness significantly increases due to the increased size of the Ag nanoplates, resulting in an increase in contact angle as expected from the Wenzel equation. This trend stops at a contact angle of $\sim 152^{\circ}$ when the surface coverage is $\sim 60 \%$ (Zone II). In both Zone I and Zone II, the water droplets do not detach from the substrates even when the substrates are inverted (Fig. S-3 in the ESM). As the sliding angle for the measurements in Zone I and Zone II is ill-defined, we assign $180^{\circ}$ as their sliding angles for convenient comparison with measurements in Zone III (below). In contrast, water droplets on the substrates with surface coverage higher than $60 \%$ can easily slide down the surfaces (with sliding angles $<30^{\circ}$ ) while the static contact angles only increase by several degrees (Zone III). As shown in the insets of Figs. 1(c) and 1(d), when the growth times are longer than 3 min, the basal surfaces of individual Ag nanoplates become rough, while the sizes of the nanoplates and the surface coverage both increase by very small percentages. Therefore, it is reasonable to conclude that the ease with which water droplets slide down the surfaces depends on the microscopic roughness of the individual nanoplates. The Ag nanoplate/GaAs composite surfaces with both macroscopic (on the micrometer and/or submicrometer scale) and microscopic (on the nanometer scale) roughness are an analog of the surfaces of lotus leaves, on which water droplets usually exhibit high contact angles and low sliding angles [14].

In order to confirm the effect of microscopic roughness of the individual nanoplates on the superhydrophobicity, we grew Ag nanoplates with very rough surfaces through reaction with $\mathrm{AgNO}_{3}$ solution with a low concentration of 0.1 mol/L [48]. Figure 3 shows a series of SEM images of the Ag products formed at different growth times. The Ag nanoplates have thicknesses of around $50 \mathrm{~nm}$ and rough basal surfaces regardless of their size. Surface coverage of Ag nanoplates on the GaAs substrates increased with growth time (Fig. 3(e)), similar to that observed above and shown in Fig. 1(e). The hydrophobic properties of the GaAs substrates covered with rough Ag nanoplates, summarized in Fig. 4, can be illustrated by plotting the dependence of the contact angle and sliding angle of water droplets on surface coverage. The use of surface coverage of Ag nanoplates, rather than growth

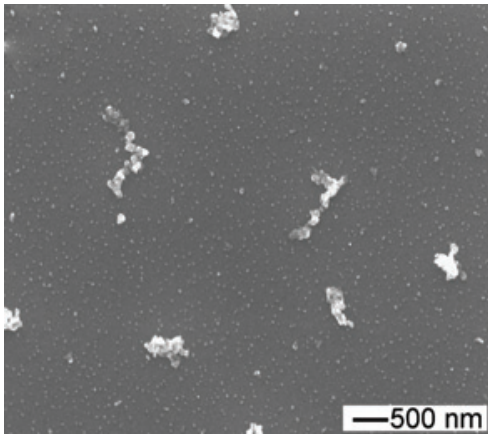

(a)

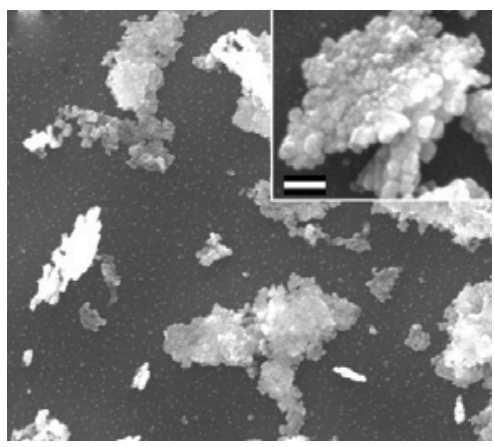

(c)

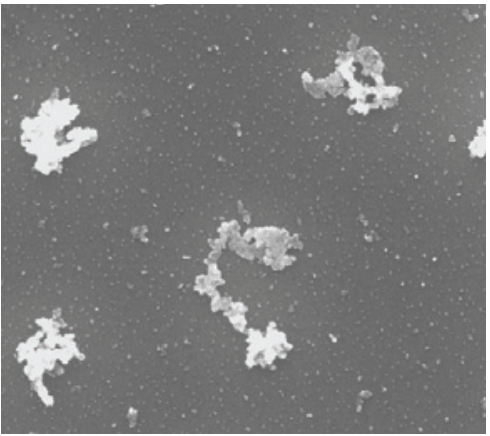

(b)

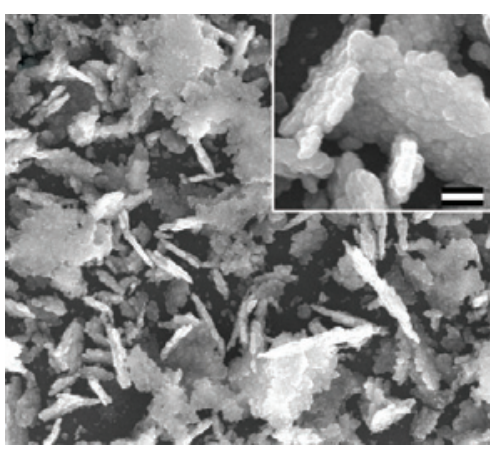

(d)

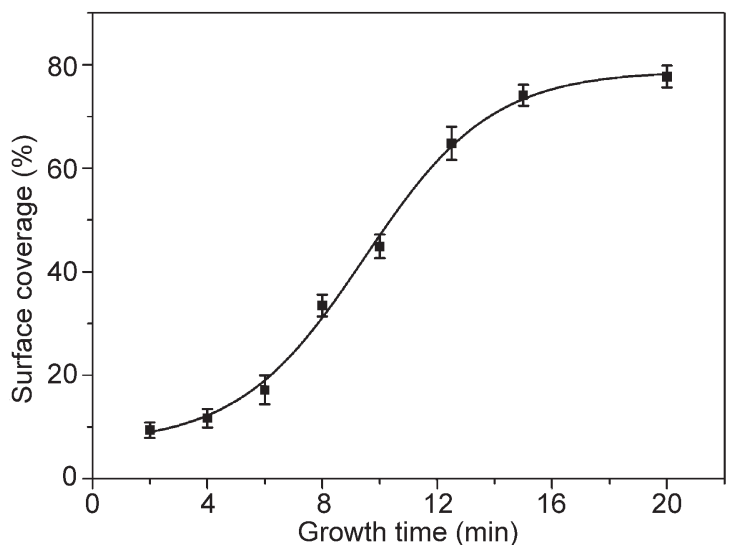

(e)
Figure 3 (a)-(d) SEM images of Ag nanoplates grown on GaAs substrates by reaction with a $0.1 \mathrm{~mol} / \mathrm{L} \mathrm{AgNO}_{3}$ solution for different times: (a) $2 \mathrm{~min}$; (b) $6 \mathrm{~min}$; (c) $8 \mathrm{~min}$; and (d) $15 \mathrm{~min}$. (e) Dependence of the surface coverage on the growth time. The scale bar in (a) also applies to (b)-(d). The scale bar in the inset of (c) is $100 \mathrm{~nm}$ and that in the inset of (d) is $250 \mathrm{~nm}$ 


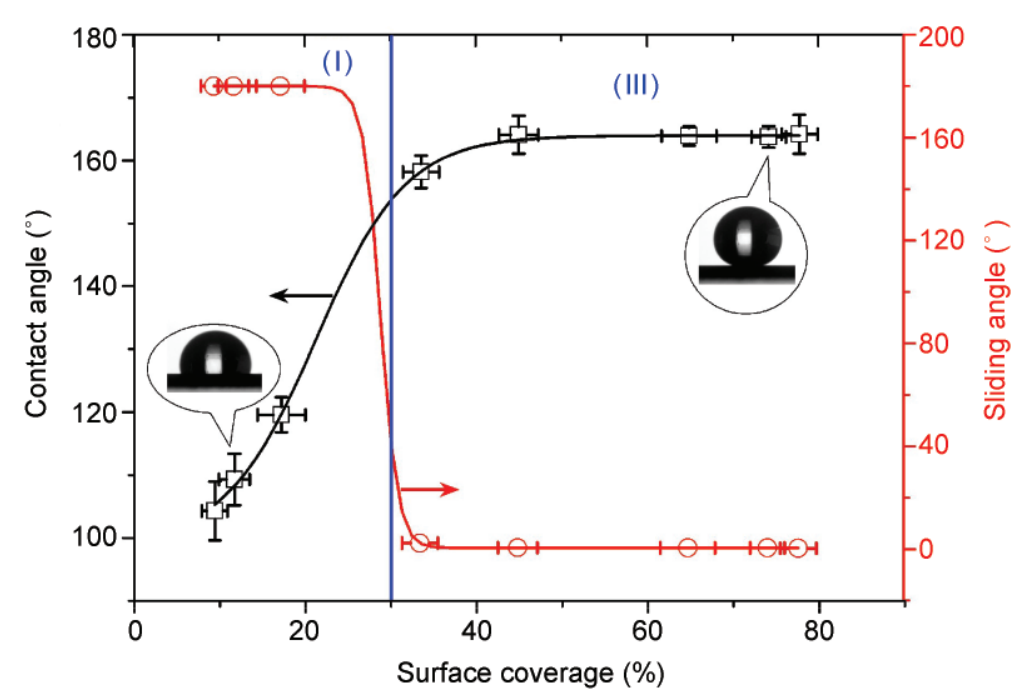

Figure 4 Relationship between apparent contact angles and sliding angles of water droplets on the surfaces shown in Fig. 3 and the surface coverage represented in Fig. 3(e). The insets show optical images of water droplets on the as-synthesized surfaces

time, allows us to directly compare the effects of varying the dimensions and densities of different Ag nanoplates on the hydrophobicity because reactions with $\mathrm{AgNO}_{3}$ solutions having different concentrations occur on different time scales. Similar to the results shown in Fig. 2, the contact angles of water droplets on the substrates covered with rough $\mathrm{Ag}$ nanoplates increase montonically up to $\sim 160^{\circ}$ with the increase in surface coverage from 0 to $\sim 30 \%$ (Zone I). The water droplets do not detach from the substrates even when the substrates are inverted. Once the surface coverage is higher than a critical value, i.e., $\sim 30 \%$, water droplets can easily slide off the substrates with very small sliding angles of $<2^{\circ}$, while the contact angles of the water droplets remain essentially constant at $\sim 164^{\circ}$ regardless of the surface coverage (Zone III). The absence of a transition state (high contact angle and sliding angle) analogous to Zone II in Fig. 2 indicates that the microscopic roughness of individual Ag nanoplates does indeed significantly lower the adhesion between water droplets and the as-fabricated substrates when the sizes of the Ag nanoplates are sufficiently large.

\section{Discussion}

A comparison of the results shown in Figs. 2 and 4 clearly shows that three different hydrophobic states (i.e., relatively low contact angle $\left(100^{\circ}-150^{\circ}\right)$ and high adhesion between water droplets and substrates (Zone I); high contact angle $\left(>150^{\circ}\right)$ and high adhesion (Zone II); and high contact angle $\left(>150^{\circ}\right)$ and low adhesion (small sliding angle) (Zone III)) can be easily achieved by simply controlling the growth time and the concentration of $\mathrm{AgNO}_{3}$ solution during the preparation of the Ag nanoplate/GaAs composite surfaces. In Zone I, where surface coverage is less than $30 \%$, the wettability is dominated by the Wenzel wetting state (Figs. 5(a) and 5(b)) for the substrates with both flat and rough Ag nanoplates. In this case, water easily penetrates into the gaps between $\mathrm{Ag}$ nanoplates to wet both the GaAs wafer and the Ag nanoplates because of the small sizes of the Ag nanoplates. The complete contact with the solid surfaces leads to strong adhesion between water droplets and the substrates. In addition, the apparent contact angles of water droplets on substrates in Zone I are consistent with the values (blue stars in Fig. 2) calculated according to the Wenzel equation:

$$
\cos \theta^{*}=r \cos \theta
$$

where $\theta^{*}$ is the apparent static contact angle; $\theta$ represents the Young's contact angle of SAMs of HDT molecules on a flat surface; and $r$ is the surface roughness, which is defined as the ratio of the actual surface area of the solid to its planar projection. The value of $\theta$ is taken as $102.6^{\circ}$ as determined from Fig. S-2(a) in the ESM. For the substrates covered with rough Ag nanoplates, $r$ and $\theta^{*}$ are difficult to calculate precisely because of the wide size distribution of the Ag nanoplates. The similarity in macroscopic hydrophobic behavior of the GaAs substrates when covered with either smooth or rough Ag nanoplates in Zone I indicates that the secondary nanometer scale roughness of the basal surfaces of individual Ag nanoplates does not significantly influence the macroscopic wetting behavior of water droplets when the sizes of the Ag nanoplates are smaller than a critical value. This critical size corresponds to a surface coverage of $\sim 30 \%$. The macroscopic surface roughness is low in Zone I.

\section{国㬎: Springer}


The contact angles computed by Eq. (2) deviate dramatically from the measured values for surface coverage higher than 30\% (Fig. 2). This discrepancy indicates that the hydrophobic behaviors in Zone II and Zone III no longer obey the Wenzel equation; they are instead dominated by the Cassie composite state, i.e., some air pockets are trapped underneath water droplets (Figs. 5(c) and 5(d)). Sitting on air pockets leads to water droplets on the surface exhibiting superhydrophobicity with apparent contact angles higher than $150^{\circ}$ [46]. According to the Cassie-Baxter equation:

$$
\cos \theta^{*}=-1+\phi_{\mathrm{s}}(1+\cos \theta)
$$

the fraction of solid in contact with liquid, $\phi_{\mathrm{s}}$, can be estimated as $\sim 15 \%$ for the water droplets in Zone II for the substrates covered with smooth Ag nanoplates by substituting $\theta^{*}$ and $\theta$ with $152^{\circ}$ and $102.6^{\circ}$ (see above), respectively. That this number is less than unity indicates that the surfaces of only some nanoplates are fully wetted by water when the sizes of the nanoplates are larger than a critical value (for example, $290 \mathrm{~nm}$ for the samples shown in Fig. 2) and the surface coverage is higher than $\sim 30 \%$. Figure 5(c) shows a schematic drawing of a so-called Cassie impregnating wetting state, which usually generates high contact angles and strong adhesion as reflected by the measurements in Zone II. The pinning of water droplets in some of the grooves formed between large flat Ag nanoplates ensures that they remain on the Ag nanoplate/GaAs surface even when the substrate is tilted or inverted. When the basal surfaces of the large Ag nanoplates become rough as shown in Fig. 3, the Ag nanoplate/ GaAs composite surfaces have roughness at both macroscopic and microscopic levels and behave in a similar way to lotus leaves, on which water droplets exhibits very high contact angles and very small sliding angles. This superhydrophobic behavior is a special Cassie state, i.e., the Cassie nonwetting state, which is consistent with the measurements in Zone III shown in Figs. 2 and 4. Figure 5(d) illustrates the microscopic contact between water droplets and the
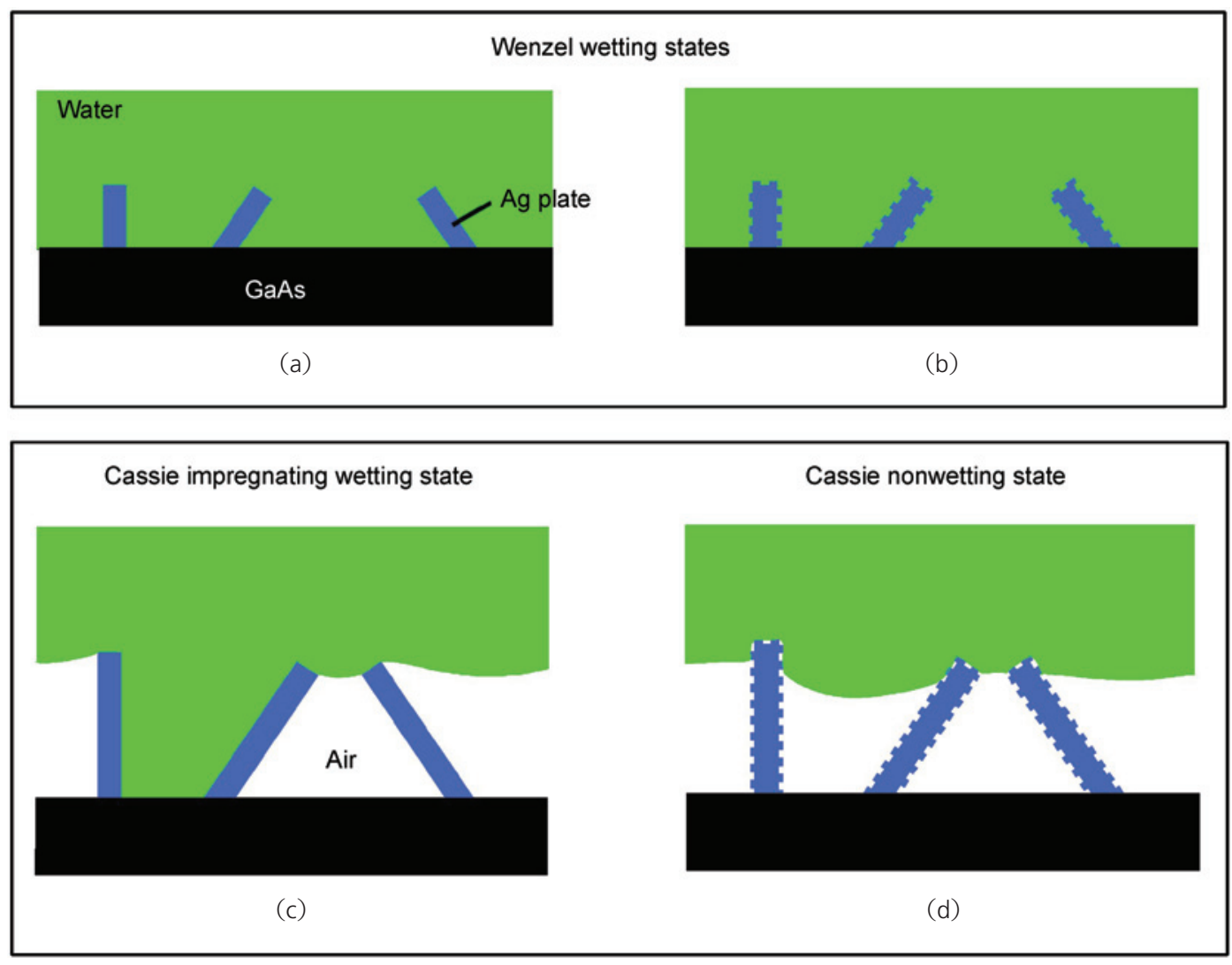

Figure 5 A schematic illustration of the contacts between water and the Ag nanoplate/GaAs composite surfaces on a microscopic scale. (a), (b), (c), (d) correspond to the hydrophobic states of Zone I in Fig. 2, Zone I in Fig. 4, Zone II in Fig. 2, and Zone III in both Figs. 2 and 4, respectively 
composite surface, which differs from the Cassie impregnating wetting state shown in Fig. 5(c). In this case, essentially no water penetrates into the gaps between the rough Ag nanoplates so that there is minimal adhesion to retain water droplets when the substrate is tilted.

In addition to the explanation shown in Fig. 5 based on the microscopic wetting between water and the Ag nanoplates (as well as the GaAs substrates), the diversity of hydrophobic states can also be understood when the Gibbs energy curve for a water droplet on an Ag nanoplate/GaAs composite surface is characterized by multiple minima (Fig. 6(a)) [45, 47]. The three local minima correspond to the three wetting states observed in this work, i.e., Wenzel wetting state for Zone I, Cassie impregnating wetting state for Zone II, and Cassie nonwetting state for

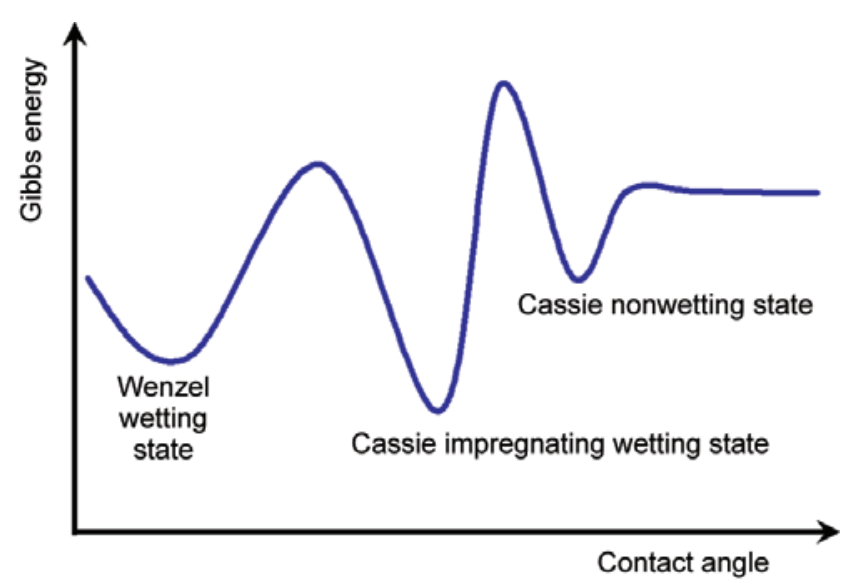

(a)

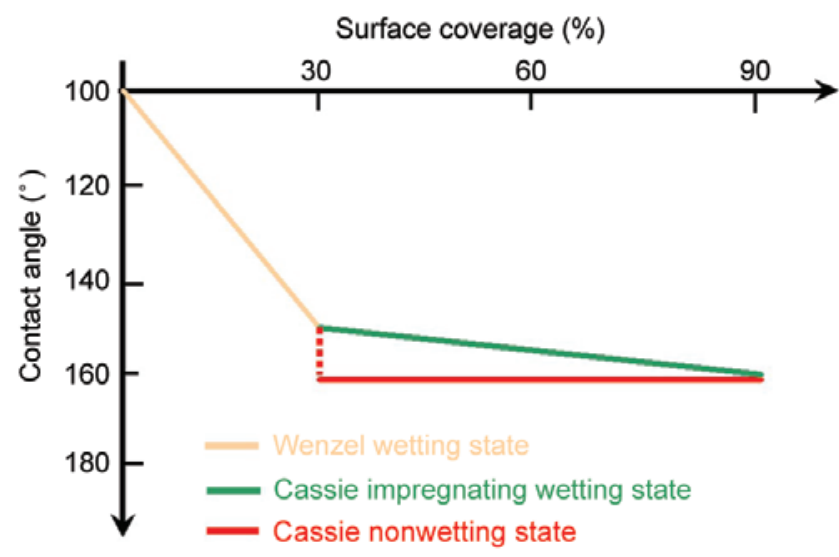

(b)

Figure 6 (a) Qualitative illustration of the dependence of the Gibbs energy on the apparent contact angle for water droplets on the $\mathrm{Ag}$ nanoplate/GaAs composite surfaces; (b) Hydrophobic states of a water droplet as a function of contact angle and surface coverage of high aspect ratio Ag nanoplates as shown in Figs. 1 and 3
Zone III. For a particular GaAs substrate covered with Ag nanoplates, a water droplet on the substrate can spontaneously minimizes its Gibbs energy to one of the three local minima, resulting in a stable apparent contact angle. The measured contact angles for the samples shown in Figs. 1-4 are approximated in the chart shown in Fig. 6(b). When the surface coverage of $\mathrm{Ag}$ nanoplates is less than $30 \%$, the thin nanoplates (thickness of $\sim 22 \mathrm{~nm}$ for the flat nanoplates in Fig. 1 and thickness of $\sim 50 \mathrm{~nm}$ for the rough nanoplates in Fig. 3) have small sizes (for example, $<290 \mathrm{~nm}$ for the flat plates). Water can easily penetrate into the gaps between nanoplates and completely wet both the surfaces of the Ag nanoplates and the GaAs substrates regardless of the roughness of individual nanoplates, resulting in the Wenzel wetting state. When the sizes of the Ag nanoplates are sufficiently large, i.e., the surface coverage is higher than $30 \%$, air in some of the grooves formed between the Ag nanoplates can be trapped underneath water droplets, leading to superhydrophobicity with apparent contact angles higher than $150^{\circ}$. For the samples with high surface coverage, both Cassie impregnating wetting state and Cassie nonwetting state are possible, depending on the nanoscale surface roughness of individual nanoplates. Water droplets on the samples with flat Ag nanoplates exhibit the Cassie impregnating wetting state, while water droplets on the samples with rough Ag nanoplates follow the behavior of the Cassie nonwetting state. Therefore, the parameters of the Ag nanoplates required to fabricate substrates with the desired hydrophobic behavior can be conveniently be determined from the chart in Fig. 6(b). By carefully controlling the growth conditions (e.g., growth time and concentration of $\mathrm{AgNO}_{3}$ solution), Ag nanoplates with predetermined parameters can be easily grown, as shown in Figs. 1 and 3. It is worthy to note that high aspect ratios of the Ag nanoplates are also important to achieve Cassie composite states. For example, thicker Ag nanoplates can be grown by reacting with $\mathrm{AgNO}_{3}$ solutions with concentration of $8 \mathrm{~mol} / \mathrm{L}$. This reaction generates Ag nanoplates with aspect ratios less than 3, although surface coverage can be up to almost $100 \%$. Measurements show that the contact angles of water droplets on

\section{国㬎: Springer}


these substrates are always less than $140^{\circ}$ (Fig. S- 4 in the ESM). This indicates that surfaces formed with assemblies of low aspect ratio Ag nanoplates do not readily exhibit superhydrophobicity.

\section{Conclusions}

Three hydrophobic states have been easily achieved on GaAs substrates covered with Ag nanoplates grown by a simple galvanic reaction. Substrates modified with small Ag nanoplates usually exhibit the Wenzel wetting states regardless of the nanometer scale roughness associated with individual nanoplates. The Cassie impregnating wetting state can occur on the surfaces of GaAs substrates covered with large flat $\mathrm{Ag}$ nanoplates. A water droplet in this state exhibits a high contact angle (i.e., $>150^{\circ}$ ) and strong adhesion to the substrate. When the GaAs substrates are coated with large rough Ag nanoplates, the Ag nanoplate/GaAs composite surfaces exhibit the "Lotus" effect, i.e., the behavior of water droplets on these surfaces obeys the Cassie nonwetting state. Surfaces with different wettabilities are expected to have applications in different areas. For example, surfaces with the Cassie impregnating wetting state can be used to transfer liquid samples without any loss [52].

\section{Acknowledgment}

The submitted manuscript has been created by UChicago Argonne, LLC, Operator of Argonne National Laboratory ("Argonne"). Argonne, a U.S. Department of Energy Office of Science Laboratory, is operated under Contract No. DE-AC02-06CH11357. Use of the Center for Nanoscale Materials and the Electron Microscopy Center for Materials Research at Argonne was supported by the U. S. Department of Energy, Office of Science, Office of Basic Energy Sciences, under Contract No. DE-AC02-06CH11357. The authors would like to thank Dr. H. H. Wang in the Materials Science Division at Argonne for his help in contact angle measurements.

Electronic Supplementary Material: Figures S-1-S-4 showing cross-sections of GaAs substrates covered with $\mathrm{Ag}$, the contact angle of a water droplet on the substrate formed after $15 \mathrm{~s}$ which is close to that of a water droplet on a flat GaAs wafer or a flat Ag film modified with SAMs of HDT molecules, the strong adhesion between water droplets and the substrate in both Zone I and Zone II, and contact angles of water droplets on $\mathrm{GaAs}$ substrates with low aspect ratio $\mathrm{Ag}$ particles, are available in the online version of this article at http://dx.doi.org/10.1007/s12274-008-8030-7 and are accessible free of charge.

\section{References}

[1] Yuan, J.; Liu, X.; Akbulut, O.; Hu, J.; Suib, S. L.; Kong, J.; Stellacci, F. Superwetting nanowire membranes for selective absorption. Nat. Nanotechnol. 2008, 3, 332336.

[2] Lahann, J. Nanomaterials clean up. Nat. Nanotechnol. 2008, 3, 320-321.

[3] Sethi, S.; Ge, L.; Ci, L.; Ajayan, P. M.; Dhinojwala, A. Gecko-inspired carbon nanotube-based self-cleaning adhesives. Nano Lett. 2008, 8, 822-825.

[4] Sun, T.; Feng, L.; Gao, X.; Jiang, L. Bioinspired surfaces with special wettability. Acc. Chem. Res. 2005, 38, 644652.

[5] Feng, X.; Jiang, L. Design and creation of superwetting/ antiwetting surfaces. Adv. Mater. 2006, 18, 3063-3078.

[6] Nosonovsky, M.; Bhushan, B. Biologically inspired surfaces: Broadening the scope of roughness. Adv. Funct. Mater. 2008, 18, 1-13.

[7] Roach, P.; Shirtcliffe, N. J.; Newton, M. I. Progress in superhydrophobic surface development. Soft Matter 2008, 4, 224-240.

[8] Li, X.-M.; Reinhoudt, D.; Crego-Calama, M. What do we need for a superhydrophobic surface? A review on the recent progress in the preparation of superhydrophobic surfaces. Chem. Soc. Rev. 2007, 36, 1350-1368.

[9] Zhang, X.; Shi, F.; Niu, J.; Jiang, Y.; Wang, Z. Superhydrophobic surfaces: From structural control to functional application. J. Mater. Chem. 2008, 18, 621633

[10] Ma, M.; Hill, R. M. Superhydrophobic surfaces. Curr. Opin. Colloid Interface Sci. 2006, 11, 193-202.

[11] Ou, J.; Perot, B.; Rothstein, J. P. Laminar drag reduction in microchannels using ultrahydrophobic surfaces. Phys. Fluids 2004, 16, 4635-4643. 
[12] Patankar, N. A. Transition between superhydrophobic states on rough surfaces. Langmuir 2004, 20, 70977102.

[13] Patankar, N. A. On the modeling of hydrophobic contact angles on rough surfaces. Langmuir 2003, 19, 1249 1253.

[14] Patankar, N. A. Mimicking the lotus effect: Influence of double roughness structures and slender pillars. Langmuir 2004, 20, 8209-8213.

[15] Feng, L.; Zhang, Y.; Xi, J.; Zhu, Y.; Wang, N.; Xia, F.; Jiang, L. Petal effect: A superhydrophobic state with high adhesive force. Langmuir 2008, 24, 4114-4119.

[16] Wenzel, R. N. Resistance of solid surfaces to wetting by water. J. Ind. Eng. Chem. 1936, 28, 988-994.

[17] Cassie, B. D.; Baxter, S. Wettability of porous surfaces. Trans. Faraday Soc. 1944, 40, 546-551.

[18] Nosonovsky, M.; Bhushan, B. Biominetic superhydrophobic surfaces: Multiscale approach. Nano Lett. 2007, 7, 26332637.

[19] Cho, W. K.; Choi, I. S. Fabrication of hairy polymeric films inspired by Geckos: Wetting and high adhesion properties. Adv. Funct. Mater. 2008, 18, 1089-1096.

[20] Cao, L.; Hu, H.-H.; Gao, D. Design and fabrication of micro-textures for inducing a superhydrophobic behavior on hydrophilic materials. Langmuir 2007, 23, 43104313.

[21] Wang, M.-F.; Raghunathan, N.; Ziaie, B. A nonlithographic top-down electrochemical approach for creating hierarchical (micro-nano) superhydrophobic silicon surfaces. Langmuir 2007, 23, 2300-2303.

[22] Winkleman, A.; Gotesman, G.; Yoffe, A.; Naaman, R. Immobilizing a drop of water: Fabricating highly hydrophobic surfaces that pin water droplets. Nano Lett. 2008, 8, 1241-1245.

[23] Love, J. C.; Gates, B. D.; Wolfe, D. B.; Paul, K. E.; Whitesides, G. M. Fabrication and wetting properties of metallic half-shells with submicron diameters. Nano Lett. 2002, 2, 891-894.

[24] Larmour, I. A.; Bell, S. E. J.; Saunders, G. C. Remarkably simple fabrication of superhydrophobic surfaces using electroless galvanic deposition. Angew. Chem. Int. Ed. 2007, 46, 1710-1712.

[25] Li, Y.; Li, C.; Cho, S. O.; Duan, G.; Cai, W. Silver hierarchical bowl-like array: Synthesis, superhydrophobicity, and optical properties. Langmuir 2007, 23, 9802-9807.

[26] Zhao, Y.; Lu, Q.; Chen, D.; Wei, Y. Superhydrophobic modification of polyimide films based on gold-coated porous silver nanostructures and self-assembled monolayers. J. Mater. Chem. 2006, 16, 4504-4509.

[27] Zhao, N.; Shi, F.; Wang, Z.; Zhang, X. Combining layerby-layer assembly with electrodeposition of silver aggregates for fabricating superhydrophobic surfaces. Langmuir 2005, 21, 4713-4716.

[28] Jiang, Y.; Wang, Z.; Yu, X.; Shi, F.; Xu, H.; Zhang, $X$. Self-assembled monolayers of dendron thiols for electrodeposition of gold nanostructures: Toward fabrication of superhydrophobic/superhydrophilic surfaces and pH-responsive surfaces. Langmuir 2005, 21, 1986-1990.

[29] Wang, S.; Feng, L.; Liu, H.; Sun, T.; Zhang, X.; Jiang, L.; Zhu, D. Manipulation of surface wettability between superhydrophobicity and superhydrophilicity on copper films. ChemPhysChem 2005, 6, 1475-1478.

[30] Mundo, R. D.; Palumbo, F.; d'Agostino, R. Nanotexturing of polystyrene surface in fluorocarbon plasmas: From sticky to slippery superhydrophobicity. Langmuir 2008, 24, 5044-5051.

[31] Gao, L.; McCarthy, T. J. A commercially available perfectly hydrophobic material. Langmuir 2007, 23, 9125-9127.

[32] Xie, Q.; Fan, G.; Zhao, N.; Guo, X.; Xu, J.; Dong, J.; Zhang, L.; Zhang, Y.; Han, C. C. Facile creation of a bionic super-hydrophobic block copolymer surface. Adv. Mater. 2004, 16, 1830-1833.

[33] Balu, B.; Breedveld, V.; Hess, D. W. Fabrication of "rolloff" and "sticky" superhydrophobic cellulose surfaces via plasma processing. Langmuir 2008, 24, 4785-4790.

[34] Jin, M.; Feng, X.; Feng, L.; Sun, T.; Zhai, J.; Li, T.; Jiang, L. Superhydrophobic aligned polystyrene nanotube films with high adhesive force. Adv. Mater. 2005, 17, 19771981.

[35] Zhai, L.; Cebeci, F. C.; Cohen, R. E.; Rubner, M. F. Stable superhydrophobic coating from polyelectrolyte multilayers. Nano Lett. 2004, 4, 1349-1353.

[36] Namavar, F.; Cheung, C. L.; Sabirianov, R. F.; Mei, W. -N.; Zeng, X. C.; Wang, G.; Haider, H.; Garvin, K. L. Lotus effect in engineered zirconia. Nano Lett. 2008, 8, 988996.

[37] Coffinier, Y.; Janel, S.; Addad, A.; Blossey, R.; Gengembre, L.; Payen, E.; Boukherroub, R. Preparation of superhydrophobic silicon oxide nanowire surfaces. Langmuir 2007, 23, 1608-1611.

[38] Ming, W.; Wu, D.; van Benthem, R.; de With, G. 
Superhydrophobic films from raspberry-like particles. Nano Lett. 2005, 5, 2298-2301.

[39] Nakajima, A.; Hashimoto, K.; Watanabe, T. Transparent superhydrophobic thin films with self-cleaning properties. Langmuir 2000, 16, 7044-7047.

[40] Pastine, S. J.; Okawa, D.; Kessler, B.; Rolandi, M.; Llorente, M.; Zettl, A.; Frechet, J. M. J. A facile and patternable method for the superface modification of carbon nanotube forests using perfluoroarylazides. J. Am. Chem. Soc. 2008, 130, 4238-4239.

[41] Zhu, L.; Xiu, Y.; Xu, J.; Tamirisa, P. A.; Hess, D. W.; Wong, C. P. Superhydrophobicity on two-tier rough surfaces fabricated by controlled growth of aligned carbon nanotube arrays coated with fluorocarbon. Langmuir 2005, 21, 11208-11212.

[42] Lau, K. K. S.; Bico, J.; Teo, K. B. K.; Chhowalla, M.; Amaratunga, G. A. J.; Milne, W. I.; McKinley, G. H.; Gleason, K. K. Superhydrophobic carbon nanotube forests. Nano Lett. 2003, 3, 1701-1705.

[43] Wang, S.; Jiang, L. Definition of superhydrophobic states. Adv. Mater. 2007, 19, 3423-3424.

[44] Lafuma, A.; Quere, D. Superhydrophobic states. Nat. Mater. 2003, 2, 457-460.

[45] Bormashenko, E.; Pogreb, R.; Stein, T.; Whyman, G.; Erlich, M.; Musin, A.; Machavariani, V.; Aurbach, D.
Characterization of rough surfaces with vibrated drops. Phys. Chem. Chem. Phys. 2008, 10, 4056-4061.

[46] McHale, G. Cassie and Wenzel: were they really so wrong? Langmuir 2007, 23, 8200-8205.

[47] Marmur, A. Soft contact: measurement and interpretation of contact angles. Soft Matter 2006, 2, 12-17.

[48] Sun, Y.; Wiederrecht, G. P. Surfactantless synthesis of silver nanoplates and their application in SERS. Small 2007, 3, 1964-1975.

[49] Sun, Y. Direct growth of dense, pristine metal nanoplates with well-controlled dimensions on semiconductor substrates. Chem. Mater. 2007, 19, 5845-5847.

[50] Sun, Y.; Yan, H.; Wu, X. Effects of visible and synchrotron $X$-ray radiation on the growth of silver nanoplates on n-GaAs wafers: A comparative study. Appl. Phys. Lett. 2008, 92, 183109.

[51] Sun, Y.; Yan, H.; Wiederrecht, G. P. Comparative study on the growth of silver nanoplates on GaAs substrates by electron microscopy, synchrotron X-ray diffraction, and optical spectroscopy. J. Phys. Chem. C 2008, 112, 8928-8938.

[52] Hong, X.; Gao, X.; Jiang, L. Application of superhydrophobic surface with high adhesive force in no lost transport of superparamagnetic microdroplet. J. Am. Chem. Soc. 2007, 129, 1478-1479. 